\title{
Effect of sensory substitution on suture-manipulation forces for robotic surgical systems
}

\author{
Masaya Kitagawa, MS \\ Daniell Dokko, BS ${ }^{a}$ \\ Allison M. Okamura, $\mathrm{PhD}^{\mathrm{a}}$ \\ David D. Yuh, MD ${ }^{\text {b }}$
}

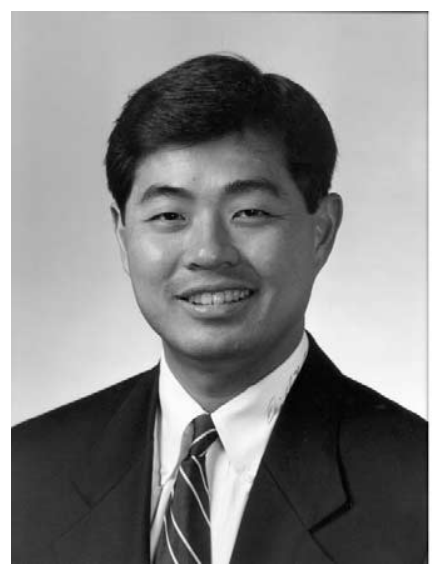

Dr Yuh
Objectives: Direct haptic (force or tactile) feedback is not yet available in commercial robotic surgical systems. Previous work by our group and others suggests that haptic feedback might significantly enhance the execution of surgical tasks requiring fine suture manipulation, specifically those encountered in cardiothoracic surgery. We studied the effects of substituting direct haptic feedback with visual and auditory cues to provide the operating surgeon with a representation of the forces he or she is applying with robotic telemanipulators.

Methods: Using the robotic da Vinci surgical system (Intuitive Surgical, Inc, Sunnyvale, Calif), we compared applied forces during a standardized surgical knot-tying task under 4 different sensory-substitution scenarios: no feedback, auditory feedback, visual feedback, and combined auditory-visual feedback.

Results: The forces applied with these sensory-substitution modes more closely approximate suture tensions achieved under ideal haptic conditions (ie, hand ties) than forces applied without such sensory feedback. The consistency of applied forces during robot-assisted suture tying aided by visual feedback or combined auditory-visual feedback sensory substitution is superior to that achieved with hand ties. Robot-assisted ties aided with auditory feedback revealed levels of consistency that were generally equivalent or superior to those attained with hand ties. Visual feedback and auditory feedback improve the consistency of robotically applied forces.

Conclusions: Sensory substitution, in the form of visual feedback, auditory feedback, or both, confers quantifiable advantages in applied force accuracy and consistency during the performance of a simple surgical task.

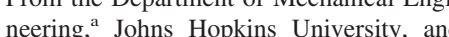
the Division of Cardiac Surgery, ${ }^{\mathrm{b}}$ Johns Hopkins Medical Institutions, Baltimore, Md.

Supported by the National Science Foundation (grant No. EEC9731478) and a gift from Ray and Jean Oglethorpe, as well as by the National Institutes of Health (R01 EB00204) and the Whitaker Foundation (RG-02-911).

Received for publication Oct 27, 2003; revisions received Feb 8, 2004; accepted for publication May 20, 2004.

Address for reprints: David D. Yuh, MD, Division of Cardiac Surgery, The Johns Hopkins Hospital, 600 North Wolfe St, Blalock 618, Baltimore, MD 21287-4618 (E-mail: dyuh@csurg.jhmi.jhu.edu)

J Thorac Cardiovasc Surg 2005;129:151-8

$0022-5223 / \$ 30.00$

Copyright (C) 2005 by The American Association for Thoracic Surgery

doi:10.1016/j.jtcvs.2004.05.029

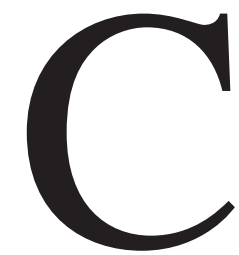

omputer-augmented, teleoperated robotic surgical systems have enhanced the ability of surgeons to perform minimally invasive operations by scaling down motions and providing additional degrees of freedom to endoscopic instrument tips. The computer interface between the surgeon and the instrument tips has now provided fine and dextrous manipulations not previously afforded by traditional manually actuated thoracoscopic instruments.

Thousands of laparoscopic surgical procedures have been performed worldwide with teleoperated robotic surgical systems. ${ }^{1}$ Clinical successes with these systems in the cardiothoracic surgical arena, however, have lagged behind for several reasons. First, the rigidity of the thoracic cage, restricted port access imposed by bony chest wall structures (ie, ribs and sternum), and continuous cardiac motion have raised significant technical challenges not found in the relatively expansile and static environment presented by the abdominal cavity. Second, the potential consequences of surgical errors or excessive delays with cardiac operations in the context of 

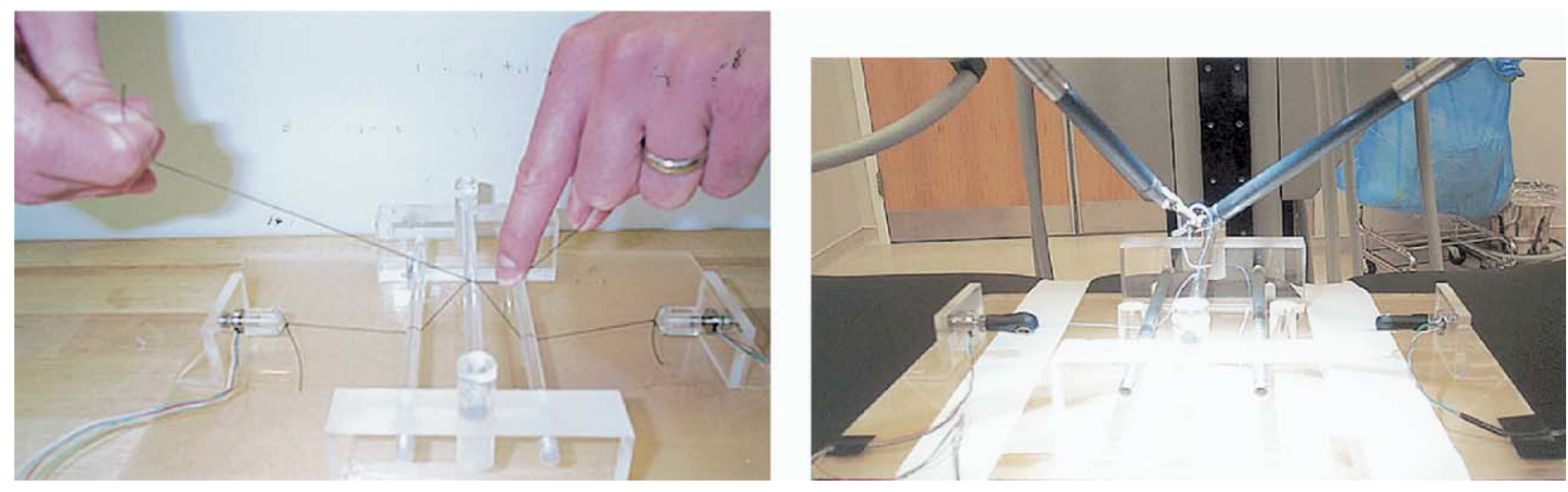

Figure 1. Left, The TMD with execution of hand suture tying. Right, The TMD during robot-assisted suture tying.

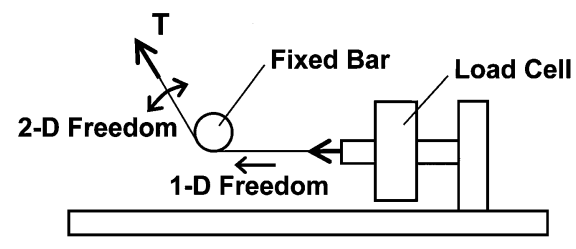

Figure 2. Side view of the TMD developed in our laboratory. The pulling force in 2 dimensions is resolved in the direction parallel to the axis of the load cell. This design allows users to perform the task in a natural way, even though the measurement device has only 1 degree of freedom.

limited exposure have given many cardiothoracic surgeons pause in applying it to their own practices. Finally, the lack of haptic (force and tactile) feedback to the surgeon might be a significant handicap in performing the technically intricate surgical tasks on delicate tissues inherent in cardiac surgical operations. ${ }^{2}$ For example, appropriate applied forces are critical in creating surgical knots that are firm enough to hold but that do not break delicate sutures or damage tissue. The clinical importance of haptic feedback in the satisfactory performance of robot-assisted surgical tasks is controversial, however, because some surgeons believe that the lack of such sensory feedback can be adequately compensated for with the use of visual queues, such as local tissue deformation with retraction or needle insertion.

A teleoperated robotic surgical system under clinical investigation is the da Vinci System (Intuitive Surgical, Inc, Mountain View, Calif). Although cardiothoracic surgeons have used this system to successfully perform coronary arterial bypass grafting, ${ }^{3,4}$ mitral valve repair, ${ }^{5,6}$ atrial septal defect closures, ${ }^{7}$ and biventricular pacemaker lead placements, ${ }^{8}$ they have generally found robot-assisted minimally invasive operations to be more time consuming than conventional open approaches. The reasons behind this observation are undoubtedly multifactorial. However, our recent

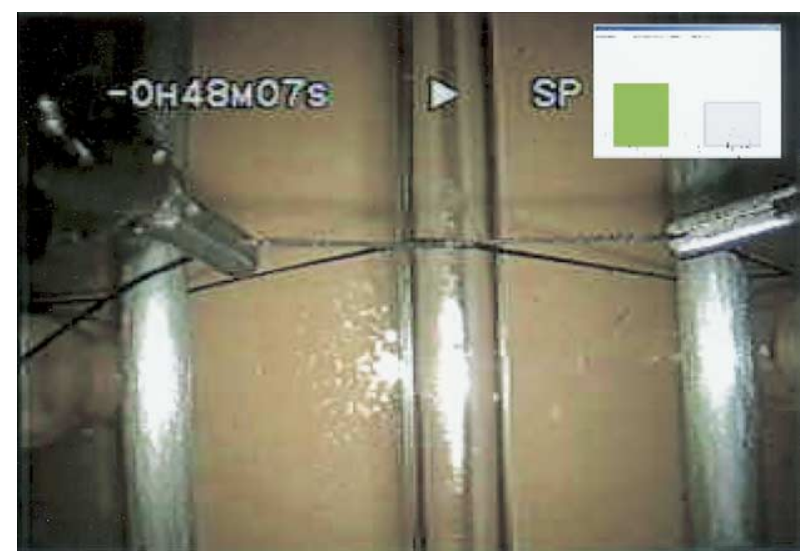

Figure 3. Visual sensory substitution of applied forces during da Vinci robot-assisted suture tying as seen by the surgeon through the da Vinci system master console. Note the visual sensory substitution representing applied suture tensions in the form of a picture-in-picture graphic in the upper right corner of the display. Two colored bars resized their height and shade according to the measured tension at the corresponding hand; green shading signals ideal suture tension.

preliminary work suggests that significant improvement in the proficient performance of robot-assisted surgical tasks is contingent on the development of haptic feedback, ${ }^{9}$ presumably because force and tactile sensing are critical to the precision and accuracy of suture ties, dissection, and tissue manipulation.

Unfortunately, complete haptic feedback is not currently available for robotic surgical systems. A provisional construct to approximate true haptic feedback is sensory substitution. Sensory substitution is the replacement of direct tactile or force feedback with other sensory modalities, specifically visual feedback (VF), auditory feedback (AF), or both, to provide the operating surgeon with a representation of the forces he or she is applying with robotic telemanipulators. It has been shown in the teleoperation 
literature that sensory-substitution feedback does enhance the ability of an operator to sense the environment and control the robot. ${ }^{10-13}$

Until now, the problems encountered with the lack of force feedback have only been described anecdotally. In these studies we seek to address the central question of whether haptic feedback has a legitimate role in robotassisted surgical systems. Here we describe our approach toward quantifying the effects of several sensory-substitution scenarios on suture-manipulation forces.

\section{Methods}

The experimental design is predicated on 3 hypotheses:

Hypothesis 1 (improved approximation of robotically applied forces to forces applied with ideal haptic feedback): The magnitude of robotic forces applied with any force-feedback method more closely approximates the suture tensions achieved under ideal haptic feedback conditions than robotic forces applied without feedback. The manual suture tension represents tensions achieved with hand ties, an ideal haptic feedback condition. We will seek to show that applied robotic forces more closely approximate tensions incurred during hand ties with substitutive feedback than without it.

Hypothesis 2 (comparable consistency of robotically applied forces compared with force applied with ideal haptic feedback): The consistency of applied forces during robot-assisted ties aided by sensory substitution is equivalent or superior to that achieved under ideal haptic feedback conditions. If this is true, the coefficients of variance $(\mathrm{CVs})$ of applied forces with robot assistance supplemented with sensory feedback will be less than or equivalent to the CVs obtained with hand ties. We will determine whether the consistency of force magnitudes applied with the robot in conjunction with sensory substitution is comparable with that achieved with ideal haptic feedback.

Hypothesis 3 (improvement in consistency of robotically applied forces): Sensory feedback improves the consistency of robotically applied forces. We will substantiate this claim by showing that the CVs of robotically applied forces with sensory substitution are less than those observed without sensory feedback.

\section{Human Subjects}

Five surgeons, 3 attending cardiac surgeons and 2 postgraduate year VIII cardiothoracic residents (Division of Cardiac Surgery, The Johns Hopkins Hospital, Baltimore, Md), performed hand and robot-assisted surgical knot ties. The attending surgeons and residents each had approximately 1 hour of experience with robotassisted suture tying. These studies were performed under a protocol titled "Haptic Sensing, Feedback, and Augmentation for Robot-Assisted Minimally Invasive Surgery," which was approved by the Johns Hopkins University Institutional Review Board on February 12, 2002.

\section{Da Vinci Surgical Robotic System}

We used the da Vinci Surgical System (Intuitive Surgical, Inc) as our teleoperated robotic platform. The da Vinci computerenhanced instrumentation system consists of 3 major compo- nents: an input device (surgeon's console), a computer interface, and an output device (manipulator).

\section{Suture Tension Measurement Device}

The tension applied to sutures during the first throw of a surgical suture knot by the left and right hands or robotic instruments was measured with a tension measurement device (TMD) developed in our laboratories (Figures 1 and 2). This device consists of two 1-degree-of-freedom Entran load cells (Entran Devices, Inc, Fairfield, NJ) tied to sutures and aluminum alloy bars used to orient the applied forces in a direction parallel to the axes of the load cells (Figure 2). An acrylic center bar provided a consistent pliable fulcrum across which the knots were tied, approximating a consistent tissue medium. The force resolution of our TMD is \pm 0.09 $\mathrm{N}$. The device design permitted users to perform the suture-tying task in a natural way while recording suture tensions.

\section{Sensory Substitution}

We measured the tension applied to sutures with the TMD during the first throw of a surgical suture knot tied with a da Vinci robot under 4 different sets of force-feedback conditions. The first condition involved no sensory feedback. The second condition included an AF mode, which provided a single tone when the magnitude of the applied tension reached the manual tension. The manual tensions for each suture material are defined as the average tensions incurred during hand ties (ideal haptic feedback condition) performed by the surgical residents and attending staff. ${ }^{9} \mathrm{AF}$ did not differentiate forces exerted by the left versus right handinstrument. The maximum force from either hand-instrument was used in comparisons against the manual tension. The third condition incorporated a VF mode, which provided a graphic display of the force levels. Two colored bars resized their height and shade according to the measured tension at the corresponding hand (Figure 3). The fourth condition incorporated combined auditoryvisual feedback (AVF) methods, whereby AF and VF, as described above, were provided simultaneously to the user.

Six different suture materials (varying by type and size) typically used in general and cardiac operations were used in these tying exercises: 2-0 silk and 2-0 Ti-Cron (Sherwood-Davis \& Geck, St Louis, Mo) and 4-0, 5-0, 6-0, and 7-0 Prolene sutures (Ethicon, Inc, Somerville, NJ). Suture tension measurements for each tie obtained with the TMD were recorded for each surgeon. Five ties with each suture material, for a total of 30 throws, were recorded for each surgeon under each force-feedback condition. Data for each surgeon were expressed as the average applied tension for each suture material incurred during hand ties (an ideal haptic feedback condition) and robot-assisted ties under each of the 4 force-feedback conditions defined above (Figure 4). Although data were acquired for both hands-instruments, only the dominant side (the right side for all subjects) was used in the data analysis; each subject performed one-hand ties, modulating the applied force primarily with the dominant hand. The subjects were instructed to aim for consistency and accuracy in applied forces in performing the ties rather than speed of completion and to hold the throw for 3 seconds at the desired tension level. Each suture was replaced after 5 ties to avoid the effects of suture material fatigue. In the few events of suture breakage, generally with robot-assisted ties, the tie was repeated with new suture material. Force data with 


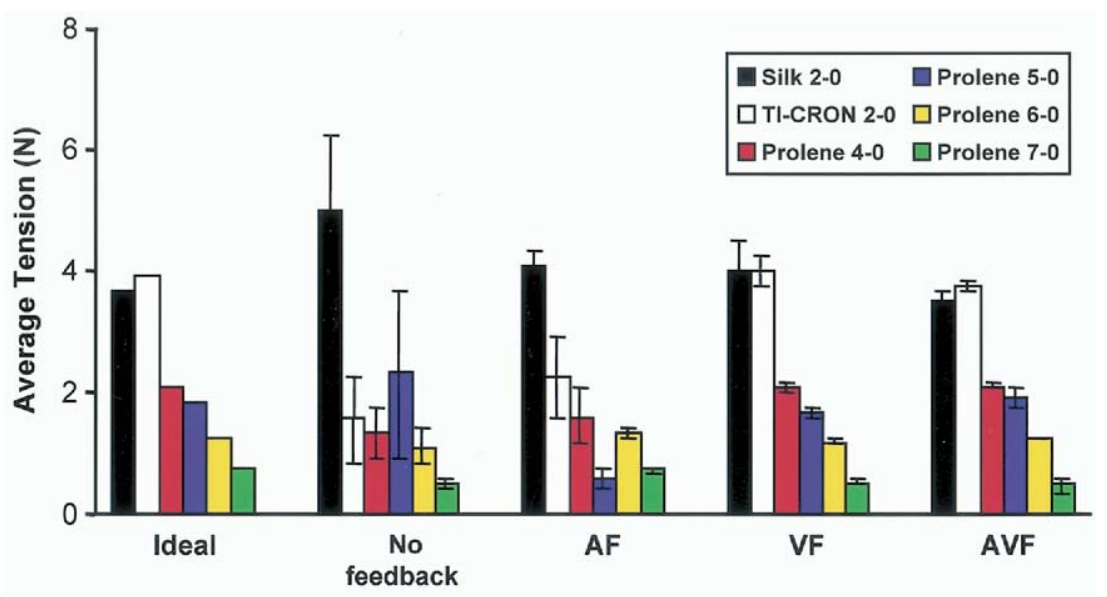

Figure 4. Example suture tension data summary for a single subject (resident surgeon, right handed). Average applied suture tensions (in newtons) measured with the TMD are shown for 5 suture ties, with each suture type under each force-feedback condition. Ideal conditions represent tensions incurred with manual ties (ideal haptic feedback). SD bars are also shown.

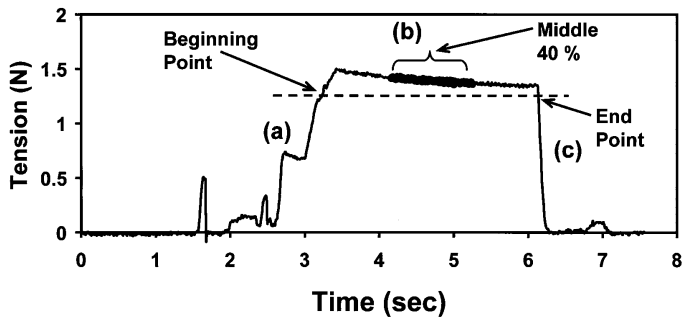

Figure 5. Tension data plotted against time for each suture throw. The data are segmented into 3 areas: (a), increasing tension; (b), holding tension; and (c), decreasing tension. The average of forces in the middle $40 \%$ of the holding region is used in data analysis.

suture breakage were not used in the analysis because it was difficult to discriminate whether the suture broke from excessive applied tension or mechanical suture damage imparted by the robot instrumentation.

\section{Data Segmentation}

The data obtained from the TMD were force applied to the suture for the left- and right-hand instruments. The tension data were plotted against time for each run (one run is shown in Figure 5). The graph consists of 3 active intervals: (1) increasing tension, (2) holding tension, and (3) decreasing tension. The holding interval was automatically segmented from the other 2 intervals at points that were $90 \%$ of the maximum tension measured during each run. By considering only data within $90 \%$ of the maximum tension, we only include data during the period that the subject intends to hold the knot. The middle $40 \%$ of the holding interval was the only portion of the data included in the calculation of the average applied tension for each run. The middle $40 \%$ was selected to avoid the force fluctuations near the start and end of the holding period. Manual examination of the data was used to select the $90 \%$ and $40 \%$ regions; these numbers were used to automate the data analysis process.

\section{Statistical Analysis}

For each of the 3 hypotheses described, the analysis of variance test, the Dunnett test, and the Duncan multiple range test were used for data analysis. We used the following linear statistical model: $\mathrm{y}_{\mathrm{ijkl}}=\mu+\alpha_{\mathrm{i}}+\beta_{\mathrm{j}}+\tau_{\mathrm{k}}+(\alpha \beta)_{\mathrm{ij}}+\epsilon_{\mathrm{ijkl}}$, where $\mathrm{y}_{\mathrm{ijkl}}$ is each observation, $\mu$ is the overall mean effect, $\alpha_{\mathrm{i}}$ is the effect of the suture type, $\beta_{\mathrm{j}}$ is the effect of the feedback methods, $\tau_{\mathrm{k}}$ is the effect of the subject, $(\alpha \beta)_{\mathrm{ij}}$ is the effect of the interaction of suture type and feedback method, and $\epsilon_{\mathrm{ijkl}}$ is random experimental error. Here we treat the subject factor as the nuisance factor.

\section{Results}

Hypothesis 1 (Improved Approximation of Robotically Applied Forces to Forces Applied with Ideal Haptic Feedback)

The first hypothesis proposes that the force magnitudes applied with any force-feedback method more closely approximate the manual suture tension than forces applied without feedback. We compared the means of the forces applied during ties executed with sensory substitution (ie, AF, VF, and AVF) with the manual tension within each suture type $(n=6)$ using the Dunnett test (Table 1). With respect to tensions achieved during AF-, VF-, and AVF-aided robotic ties versus the corresponding manual tensions achieved with hand ties, comparisons among all 5 surgeons resulted in no significant differences in the mean applied forces, except in the case of AF with 2-0 Ti-Cron suture. These results indicate that the applied forces achieved with these sensory-substitution modes did not vary significantly from the manual forces achieved with hand ties. Hence the magnitude of force applied by using these sensory-substitution modes more closely approximates the 
TABLE 1. Confidence intervals of Dunnett test results comparing the mean applied forces during ties executed with sensory substitution with the ideal tension within each suture type $(n=6)$

\begin{tabular}{|c|c|c|c|c|c|c|}
\hline \multirow[b]{2}{*}{ Ideal vs } & \multirow[b]{2}{*}{ Silk 2-0 } & \multirow[b]{2}{*}{ TI-CRON 2-0 } & \multicolumn{4}{|c|}{ Polypropylene } \\
\hline & & & $4-0$ & $5-0$ & $6-0$ & $7-0$ \\
\hline $\mathrm{AF}$ & $\begin{array}{c}-0.19 \text { to } 1.10 \\
\text { (NS) }\end{array}$ & $\begin{array}{c}-0.01 \text { to } 1.28 \\
\text { (NS) }\end{array}$ & $\begin{array}{c}-0.82 \text { to } 0.48 \\
\text { (NS) }\end{array}$ & $\begin{array}{c}-1.09 \text { to } 0.21 \\
\text { (NS) }\end{array}$ & $\begin{array}{c}-0.71 \text { to } 0.59 \\
\text { (NS) }\end{array}$ & $\begin{array}{c}-0.76 \text { to } 0.53 \\
\text { (NS) }\end{array}$ \\
\hline VF & $\begin{array}{c}-0.49 \text { to } 0.81 \\
\text { (NS) }\end{array}$ & $\begin{array}{c}-0.66 \text { to } 0.64 \\
\text { (NS) }\end{array}$ & $\begin{array}{c}-0.59 \text { to } 0.71 \\
\text { (NS) }\end{array}$ & $\begin{array}{c}-0.64 \text { to } 0.66 \\
\text { (NS) }\end{array}$ & $\begin{array}{c}-0.68 \text { to } 0.62 \\
\text { (NS) }\end{array}$ & $\begin{array}{c}-0.69 \text { to } 0.61 \\
\text { (NS) }\end{array}$ \\
\hline AVF & $\begin{array}{c}-0.60 \text { to } 0.70 \\
\text { (NS) }\end{array}$ & $\begin{array}{c}-0.52 \text { to } 0.78 \\
\text { (NS) }\end{array}$ & $\begin{array}{c}-0.58 \text { to } 0.72 \\
\text { (NS) }\end{array}$ & $\begin{array}{c}-0.63 \text { to } 0.67 \\
\text { (NS) }\end{array}$ & $\begin{array}{c}-0.63 \text { to } 0.67 \\
\text { (NS) }\end{array}$ & $\begin{array}{c}-0.71 \text { to } 0.59 \\
\text { (NS) }\end{array}$ \\
\hline
\end{tabular}

$A F$, Audio feedback; $V F$, visual feedback; $A V F$, audio and visual feedback; $N S$, not significant.

manual suture tension achieved with hand ties than forces applied without such sensory feedback, confirming hypothesis 1 .

\section{Hypothesis 2 (Comparable Consistency of Robotically} Applied Forces Compared with Forces Applied with Hand Ties)

The second hypothesis proposes that the consistency of applied forces during robot-assisted ties aided by sensory substitution is equivalent or superior to that achieved with ideal haptic feedback. We tested this by determining whether the CVs of applied forces for robot-assisted ties executed with sensory substitution are less than or equivalent to those achieved with hand ties. The CV for each suture type was analyzed $(n=6)$ by the Dunnett test (Figure 6). We compared the average $\mathrm{CV}$ of the hand ties with the average CV of the robot-assisted ties with (1) the 3 sensory-substitution modes and (2) no sensory feedback for each suture type. The average CVs obtained during robot-assisted ties with no sensory feedback were significantly greater than those obtained with hand ties for all 6 suture types, indicating less consistent applied forces with no feedback. Comparing robot-assisted ties aided by AF sensory substitution with hand ties, 3 of the 6 comparisons displayed similar average CVs (2-0 silk, 4-0 Prolene, and 7-0 Prolene); 2 comparisons revealed lesser CVs (2-0 Ti-Cron and 6-0 Prolene), reflecting more consistent applied forces; and 1 comparison revealed a greater CV (5-0 Prolene), indicating less consistency. Comparing robot-assisted ties aided by VF (Table 2) and AVF sensory substitution with hand ties, comparisons revealed significantly lesser average CVs across all 6 suture types, indicating more consistent applied forces with these sensory feedback modes. These results suggest that the consistency of applied forces during robot-assisted suture tying aided by VF or AVF sensory substitution is superior to that achieved with hand ties. Furthermore, robot-assisted ties aided with AF revealed levels of consistency that were generally equivalent or superior to those attained with hand ties, confirming hypothesis 2 .

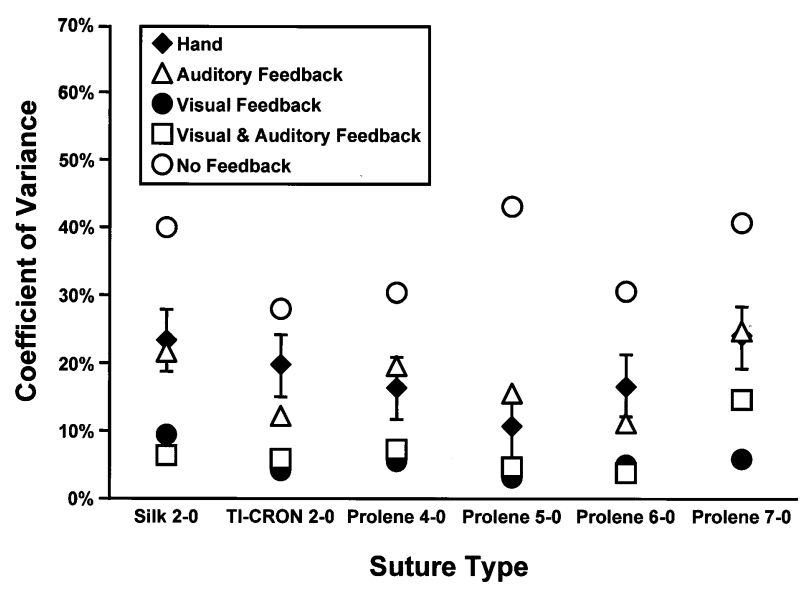

Figure 6. The average CVs of the applied forces achieved during suture tying performed by hand and with robot assistance with and without sensory substitution. Each column represents 1 of 6 different suture material types. The error bar for the hand ties (ideal haptic feedback conditions) corresponds to the critical difference for the Dunnett multiple range test.

\section{Hypothesis 3 (Improvement in Consistency of Robotically Applied Forces)}

The third hypothesis proposes that sensory feedback improves the consistency of robotically applied forces. The effect of AF and VF on the consistency of robotically applied forces in performing suture tying with the da Vinci system was examined. Correlations between suture type or subject and average CV values were not found to be statistically significant. The CVs of each of the different sensorysubstitution modes were also compared with each other, revealing no significant differences. Comparing the $\mathrm{CV}$ of robotically applied forces aided by AF sensory substitution with that of applied forces without sensory substitution revealed an improvement in consistency (ie, lesser CV) of $50.2 \%$ (Table 3); there was an $84.1 \%$ improvement observed with the aid of VF. Using the Duncan multiple range test, we also found that when VF was operative, the addition of AF did little to improve the consistency of robotically 
TABLE 2. Coefficients of variance for hand versus robotassisted ties performed with visual feedback for all suture types

\begin{tabular}{lcccrc}
\hline & & Hand & VF & \multicolumn{1}{c}{$95 \%$ Cl } & $\boldsymbol{P}$ value $^{*}$ \\
\hline Silk & $2-0$ & $22.3 \%$ & $9.5 \%$ & $8.3 \%-17.3 \%$ & $<.05$ \\
TI-CRON & $2-0$ & $19.1 \%$ & $4.3 \%$ & $10.3 \%-19.3 \%$ & $<.05$ \\
Prolene & $4-0$ & $16.1 \%$ & $5.7 \%$ & $5.9 \%-14.9 \%$ & $<.05$ \\
& $5-0$ & $10.7 \%$ & $3.2 \%$ & $3.0 \%-12.0 \%$ & $<.05$ \\
& $6-0$ & $18.0 \%$ & $5.1 \%$ & $8.4 \%-17.4 \%$ & $<.05$ \\
& $7-0$ & $25.2 \%$ & $5.5 \%$ & $15.2 \%-24.2 \%$ & $<.05$ \\
\hline
\end{tabular}

$V F$, Visual feedback; $C l$, confidence Interval.

*Dunnett test comparisons at $95 \%$ confidence level.

applied forces. Thus, VF and AF appear to improve the consistency of robotically applied forces, confirming hypothesis 3 .

\section{Discussion}

Technologic advancements in computer-assisted surgical systems have driven the expansion of minimally invasive techniques in cardiothoracic surgery. Robotic surgical systems present several advantages over conventional thoracoscopic techniques, including 3-dimensional vision, increased range of motion, tremor filtration, and motion scaling. ${ }^{14,15}$ Together, these new capabilities permit rather intricate surgical tasks to be performed in the confined and crowded environment of the thoracic cage and mediastinum. The da Vinci system has been used successfully to perform endoscopic coronary artery bypass grafting, $3,4,16$ mitral valve repairs, ${ }^{5,17-19}$ atrial septal defect closures, ${ }^{7}$ and other cardiothoracic procedures. The delicate tissues involved, the significant consequences of inadvertent tissue injury, and the manipulation of fine polypropylene sutures inherent in cardiothoracic surgery have prompted investigation into validating and incorporating force-feedback mechanisms into surgical robotic systems. Supporting the need for haptic feedback in robotic surgical systems, force feedback in teleoperated systems has been shown to improve the performance of a user in some situations. ${ }^{20,21}$ Hannaford and colleagues ${ }^{22}$ asserted that "the addition of kinesthetic force feedback is of substantial help in moving performance toward the extreme, demonstrated by the bare-handed human" in a force-sensing teleoperated system. Furthermore, Rosen and associates ${ }^{23}$ showed that bilateral telemanipulation of an endoscopic instrument returned haptic information that was lost when a surgeon manipulated soft tissues with a traditional endoscopic tool-grasper.

Ideally, haptic feedback in robotic surgical systems would be relayed directly to the surgeon's control actuators, rendering true force feedback. This capability, however, will require the application of force sensors on the endoscopic instrumentation. Our laboratories are actively pursu- ing this goal. As an interim alternative to haptic feedback, we have used sensory substitution to provide force feedback through a visual display and auditory tones. Sensory-substitution feedback appears to enhance the ability of an operator to sense the environment and control the robot. Using a peg-in hole task with a simple force-variance detection test, Massimino and coworkers ${ }^{10,12}$ showed that a combination of vibrotactile and auditory substitutions permitted task performances comparable with those performed with force feedback. In detecting a small change in force, the user performed better with the 2 combined substitutions than with force feedback alone. Benefits of redundant AF combined with haptic feedback in a virtual environment have also been reported. ${ }^{13}$ On the other hand, Massimino also concluded that redundant VF actually slowed task performances, perhaps an example of "sensory overload." Of note, none of these experiments focused on the magnitude of force generated by the user but on performance in terms of time to completion. Debus and colleagues ${ }^{11}$ conducted studies showing that vibrotactile feedback significantly lowered the mean applied force error by using a teleoperator system composed of 2 PHANTOM haptic devices (SensAble Technologies, Woburn, Mass). In these studies VF was not as effective as vibrotactile feedback. We should also add that these sensory-substitution studies were not performed in the context of surgical tasks.

Unlike this previous work, our studies focused on the effects of force feedback and the force levels and repeatability of a task directly relevant to robot-assisted surgery. We sought to quantify the differences between the performances of surgeons during a suture-manipulation task, suture knot tying, with various sensory-substitution scenarios, including no feedback, AF, VF, and AVF. We used the results from these experiments to validate 3 hypotheses addressing the utility of sensory substitution for robotassisted surgical systems.

All of the hypotheses were satisfied in that the surgeons' performance, in terms of the accuracy and consistency of applied forces, of robotic ties with force sensory substitution was at least comparable with performances during hand ties if not better. The CVs for the robotic ties under VF were consistently lower than those of the hand ties. The dominant effect of VF is evident from the observed relative effects of $\mathrm{VF}$ and AF modes. This is likely due to the fact that our subjects were provided with continuous feedback information with our VF mechanism, whereas our AF method signaled the user only when the ideal tension was reached. The initial design of the AF mechanism was to provide continuous information by varying a volume, pitch, and tone of sound. However, such continuous AF might be disruptive and confusing in an already noisy operating room environment (eg, cardiopulmonary bypass pump and hemodynamic pulse oximetery monitors). Furthermore, commu- 
TABLE 3. Comparisons of the coefficients of variance of robotically applied forces aided by each of the sensorysubstitution modes with that of robotically applied forces without sensory substitution

\begin{tabular}{lccc}
\hline \multicolumn{1}{c}{ Comparison groups } & $\begin{array}{c}\text { Difference in } \\
\text { coefficient of } \\
\text { variance }\end{array}$ & 95\% Cl & $\boldsymbol{P}$ value \\
\hline No feedback vs VF & 29.66 & 16.01 to 43.31 & $<.05$ \\
No feedback vs AVF & 28.22 & 14.57 to 41.87 & $<.05$ \\
No feedback vs AF & 17.74 & 4.09 to 31.39 & $<.05$ \\
AF vs VF & 10.48 & -2.73 to 23.69 & NS \\
AF vs AVF & 11.92 & -1.29 to 25.13 & NS \\
AVF vs VF & 1.44 & 11.1 to 13.98 & NS \\
\hline
\end{tabular}

$\mathrm{Cl}$, Confidence interval; $A F$, audio feedback; $V F$, visual feedback; $A V F$, audio and visual feedback; $N S$, not significant.

nication between assistants and the surgeon could be distracted. Therefore, one could reasonably argue that $\mathrm{AF}$ would have been as effective as VF in these experiments had a continuous feedback design been used.

Of note, during the conduct of these experiments, surgeons strongly favored VF over AF because of the availability of continuous real-time information as opposed to the discrete single-event information provided by our AF system. One of the many features to which the surgeons responded positively was the color-changing nature of the VF indicator bars. This meant that they could use the discrete nature of the color signal, detectable in the visual periphery, when they chose not to visually focus on the continuously changing height of the bars. Therefore, a visual force-feedback display in the operative field display might ultimately prove to be more ergonomic than its auditory counterpart.

Despite our rationale, the fact that only force data acquired from the dominant hands of our subjects were used in the analysis might represent a study limitation. It is quite possible that sensory substitution and haptic feedback improves the accuracy and consistency of force applied by the nondominant hand. Confirming this might, in part, support observations from some experienced surgeons that robotic surgical systems improve the dexterity of the nondominant hand.

The ultimate goal of this research was to quantitatively characterize the effects of force feedback in the form of sensory substitution on suture-manipulation forces. Although this work shows that the sensory substitution (ie, AF and VF) can provide sufficient feedback for the user to control the application of robotically applied force, true haptic (force and tactile) feedback remains a more intuitive mode for surgeons to effectively perform robot-assisted tasks. Thus, our next technical goal is to equip teleoperated robotic surgical instrumentation with force-feedback mech- anisms that would enable direct haptic feedback to the operating surgeon. In such a system, sensory substitution might take a supporting role along with direct haptic feedback. Furthermore, we need to establish whether the effects of force feedback on suture-manipulation forces actually influence surgical performance. This would be the focus of a future study with clinically relevant performance measures.

We thank Dr Christopher J. Hasser of Intuitive Surgical, Inc, and Drs Vincent L. Gott, William A. Baumgartner, Mark A. Talamini, Marc S. Sussman, T.-Y. Hsia, Torin Fitton, Stephen Cattaneo, and Randy Brown of the Division of Cardiac Surgery, Department of Surgery, and the Minimally Invasive Surgical Training Center at The Johns Hopkins Medical Institutions for their encouragement and assistance. We also acknowledge Craig M. Hooker, MPH, who provided biostatistical review of this work.

\section{References}

1. Yoshino I, Hashizume M, Shimada M, Tomikawa M, Tomiyasu M, Suemitsu R, et al. Thoracoscopic thymectomy with the da Vinci computer-enhanced surgical system. J Thorac Cardiovasc Surg. 2001; 122:783-5

2. Shennib H, Bastawisy A, Mack MJ, Moll FH. Computer-assisted telemanipulation: an enabling technology for endoscopic coronary artery bypass. Ann Thorac Surg. 1998;66:1060-3.

3. Kappert U, Cichon R, Schneider J, Gulielmos V, Tugtekin SM, Matschke K, et al. Closed-chest coronary artery surgery on the beating heart with the use of a robotic system. J Thorac Cardiovasc Surg. 2000;120:809-11.

4. Boyd WD, Rayman R, Desai ND, Menkis AH, Dobkowski W, Ganapathy $\mathrm{S}$, et al. Closed-chest coronary artery bypass grafting on the beating heart with the use of a computer-enhanced surgical robotic system. J Thorac Cardiovasc Surg. 2000;120:807-9.

5. Chitwood WR, Nifong LW. Minimally invasive videoscopic mitral valve surgery: the current role of surgical robotics. J Card Surg. 2000;15:61-75.

6. Felger JE, Nifong LW, Chitwood WR. The evolution of and early experience with robot-assisted mitral valve surgery. Surg Laparosc Endosc Percutan Tech. 2002;12:58-63.

7. Reichenspurner H, Boehm DH, Welz A, Schulze C, Zwissler B, Reichart B. 3D-video- and robot-assisted minimally invasive ASD closure using the Port-Access techniques. Heart Surg Forum. 1998;1: 104-6.

8. DeRose JJ, Ashton RC, Belsley S, Swistel DG, Vloka M, Ehlert F, Shaw R, et al. Robotically assisted left ventricular epicardial lead implantation for biventricular pacing. J Am Coll Cardiol. 2003;41: 1414-9.

9. Kitagawa M, Okamura AM, Bethea BT, Gott VL, Baumgartner WA. Analysis of suture manipulation forces for teleoperation with force feedback. In: Dohi T, Kikinis R, editors. Lecture Notes in Computer Science, vol 2488. Proceedings of the Fifth International Conference on Medical Image Computing and Computer Assisted InterventionMICCAI 2002 Sep 26-29; Tokyo, Japan. Heidelberg, Springer-Verlag; p. $155-62$

10. Massimino MJ. Improved force perception through sensory substitution. Control Eng Pract. 1995;3:215-22.

11. Debus T, Becker T, Dupont P, Jang T, Howe R. Telemanipulator and Telepresence Technology VIII Conference, vol 4570. Proceedings of SPIE (The International Society for Optical Engineering), Newton (MA), 2001 Oct 28; Bellingham (WA), SPIE.

12. Massimino MJ, Sheridan TB. Sensory substitution for force feedback in teleoperation. IFAC Symposia Series; 1992 Jun 9-11; Hague, The Netherlands. Tarrytown (NY), Pergamon Press; 1993. 
13. Richard $P$, Coiffet $P$. Human perceptual issues in virtual environments: sensory substitution and information redundancy. In: Robot and $\mathrm{Hu}-$ man Communication. Proceedings of the IEEE International Workshop; Tokyo, 1995 Jul 5-7; Piscataway (NJ), IEEE; 1995.

14. Schneeberger E, Michler R. An overview of the Intuitive system: the surgeon's perspective. Operative Techniques Thorac Cardiovasc Surg. 2001;6:170-6.

15. Czibik G, D'Ancona G, Donias HW, Karamanoukian HL. Robotic cardiac surgery: present and future applications. J Cardiothorac Vasc Anesth. 2002;16:495-501.

16. Damiano RJ. Endoscopic coronary artery bypass grafting-the first steps on a long journey. J Thorac Cardiovasc Surg. 2000;120: 806-7.

17. Grossi EA, Lapietra A, Applebaum RM, Ribakove GH, Galloway AC, Baumann FG, et al. Case report of robotic instrument-enhanced mitral valve surgery. J Thorac Cardiovasc Surg. 2000;120: 1169-71.

18. Autschbach R, Onnasch JF, Falk V, Walther T, Kruger M, Schilling
LO, et al. The Leipzig experience with robotic valve surgery. $J$ Card Surg. 2000;15:82-7.

19. Chitwood WR Jr. Robotic mitral valve surgery. Heart Surg Forum. 2003;6:106-7.

20. Dennerlein JT, Martin DB, Hasser C. Force-feedback improves performance for steering and combined steering-targeting tasks. In: Proceedings of the Conference on Human Factors in Computing Systems. 2000 Apr 1-6; Hague, The Netherlands. New York: Association for Computing Machinery; 2000.

21. Dennerlein JT, Yang MC. Haptic force-feedback devices for the office computer: performance and musculoskeletal loading issues. Hum Factors. 2001;43:278-86.

22. Hannaford B, Wood L, McAffee D, Zak H. Performance evaluation of a six-axis generalized force-reflecting teleoperator. IEEE Trans Systems Man Cybernetics. 1991;21(3):620-33.

23. Rosen J, Hannaford B, MacFarlane MP, Sinanan MN. Force controlled and teleoperated endoscopic grasper for minimally invasive surgeryexperimental performance evaluation. IEEE Trans Biomed Eng. 1999; 46:1212-21. 